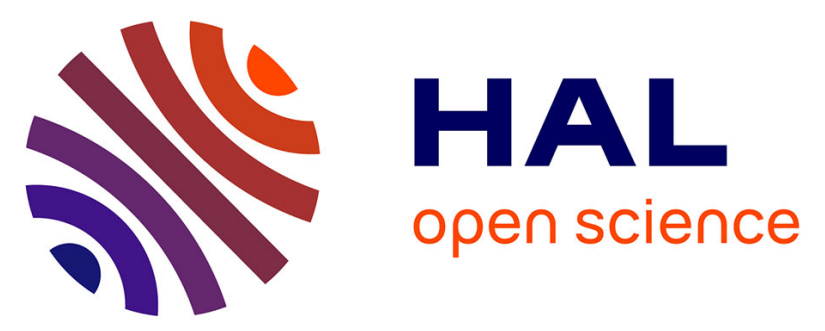

\title{
Conformation of surface-decorating dextran chains affects the pharmacokinetics and biodistribution of doxorubicin-loaded nanoparticles
}

Khairallah Alhareth, Christine Vauthier, Fanchon Bourasset, Claire Gueutin, Gilles Ponchel, Fathi Moussa

\section{To cite this version:}

Khairallah Alhareth, Christine Vauthier, Fanchon Bourasset, Claire Gueutin, Gilles Ponchel, et al.. Conformation of surface-decorating dextran chains affects the pharmacokinetics and biodistribution of doxorubicin-loaded nanoparticles. European Journal of Pharmaceutics and Biopharmaceutics, 2012, 81 (2), pp.453 - 457. 10.1016/j.ejpb.2012.03.009 . hal-03196146

\section{HAL Id: hal-03196146 \\ https://hal.science/hal-03196146}

Submitted on 12 Apr 2021

HAL is a multi-disciplinary open access archive for the deposit and dissemination of scientific research documents, whether they are published or not. The documents may come from teaching and research institutions in France or abroad, or from public or private research centers.
L'archive ouverte pluridisciplinaire HAL, est destinée au dépôt et à la diffusion de documents scientifiques de niveau recherche, publiés ou non, émanant des établissements d'enseignement et de recherche français ou étrangers, des laboratoires publics ou privés. 


\section{Conformation of surface-decorating dextran chains affects the pharmacokinetics and biodistribution of doxorubicin- loaded nanoparticles}

Khairallah Alhareth ${ }^{1,2+}$, Christine Vauthier ${ }^{2, *}$, Fanchon Bourasset $^{3}$, Claire Gueutin $^{1,2}$, Gilles Ponchel ${ }^{1,2}$, Fathi Moussa ${ }^{4, *}$

1 Univ Paris Sud, Physico-chimie, Pharmacotechnie et Biopharmacie, UMR 8612, ChâtenayMalabry, F-92296

${ }^{2}$ CNRS, Châtenay-Malabry, F-92296

3 Univ Paris Sud, Laboratoire de Pharmacie Clinique, EA 4123, Châtenay-Malabry, F-92296

${ }^{4}$ Univ Paris Sud, LETIAM, IUT d'Orsay, Orsay, F-91400

Published in: Eur J Pharm Biopharm 81 (2012) 453-457. DOI: 10.1016/j.ejpb.2012.03.009.

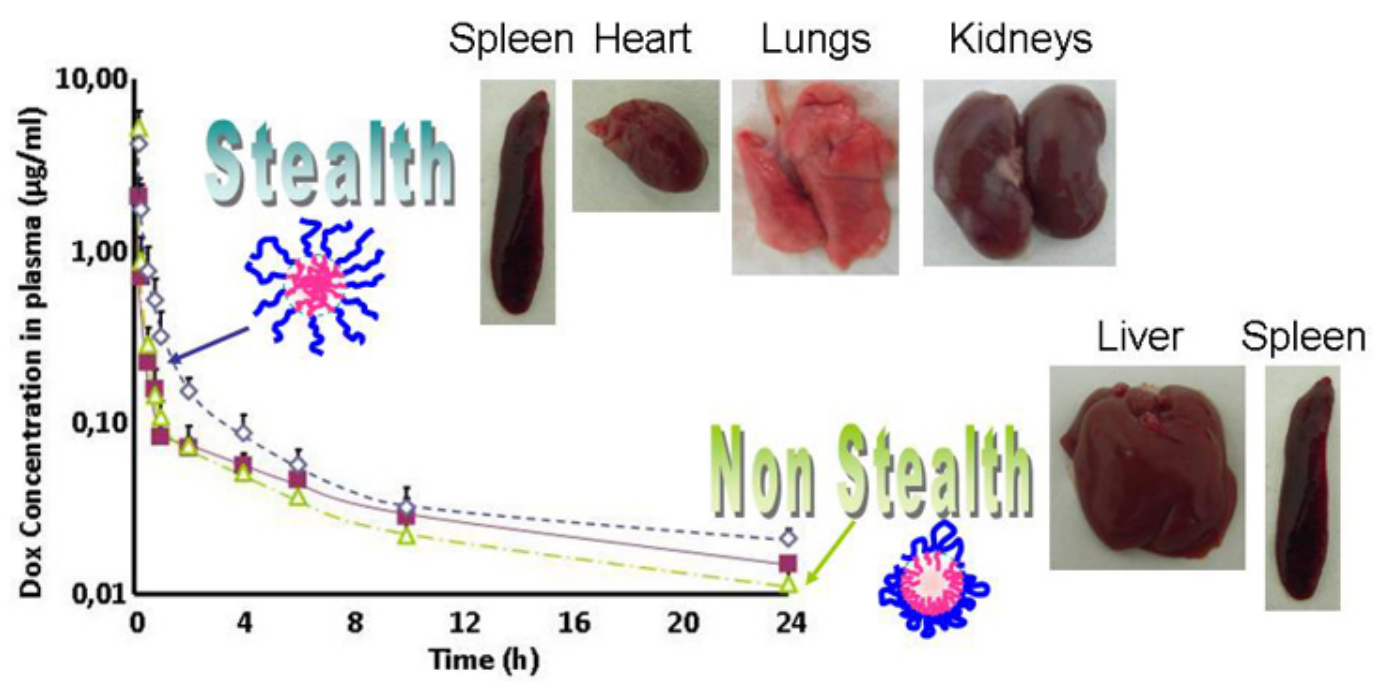

\section{${ }^{*}$ Corresponding Authors:}

Christine Vauthier, Physico-chimie, Pharmacotechnie, Biopharmacie, UMR CNRS 8612, Faculté de Pharmacie, Université de Paris Sud, 5 Rue J.B. Clément 92296 ChâtenayMalabry Cedex, France, christine.vauthier@u-psud.fr, Phone 331468356 03, Fax 33146 835946

Fathi Moussa, Univ Paris Sud, LETIAM, IUT d'Orsay, Orsay, F-91400 fathi.moussa@upsud.fr

+ address in 2021: Unité de Technologies Chimiques et Biologiques pour la Santé (UTCBS), UMR 8258 CNRS -U1267 INSERM, Faculté des Sciences Pharmaceutiques et Biologiques, 4 Avenue de l'Observatoire, 75270 Paris Cedex 06, France 


\section{Abstract}

Recent reports showed that subtle modifications of nanoparticle surface properties induced dramatic changes of interactions with serum proteins. The present work was aimed to investigate the effect of the conformation of dextran- chains decorated the surface of poly(alkylcyanoacrylate) (PACA) nanoparticles on the pharmacokinetic and biodistribution of a model drug associated with the nanoparticles. Doxorubicin was associated with PACA nanoparticles prepared by AEP (Dox-AEP) and RREP (Dox-RREP). Nanoparticles and the free drug (f-Dox) were injected intravenously to rats to determine the pharmacokinetic and biodistribution of doxorubicin. Curves of the pharmacokinetics showed a rapid phase of distribution followed by a slower elimination phase. Pharmacokinetic parameters of the distribution phase determined for the Dox-RREP were significantly different from those of $f$ Dox and Dox-AEP while no difference was observed in the elimination phase of the three formulations. Rats treated with Dox-RREP showed lower Dox concentrations in liver but higher concentrations in heart, lungs and kidneys compared to those treated with the other formulations. Dox-RREP exhibited a new type of stealth behavior characterized by a short circulation time and a rapid distribution in highly vascularized organs bypassing the MPS. The difference in pharmacokinetic and biodistribution observed between the drug formulated with the two types of nanoparticles was attributed to the difference in the conformation of the dextran chains stranded on the nanoparticle surface.

\section{Key words:}

Stealth nanoparticles, nanoparticle surface, dextran, polysaccharide-coating, anionic emulsion polymerization. 


\section{Introduction:}

Many types of nanoparticles were developed as drug carriers to modify tissue distribution of drugs. Among those, poly(alkylcyanoacrylate) nanoparticles (PACA-NPs) were the most studied nanoparticles [1].

The first PACA-NPs prepared by anionic emulsion polymerization (AEP) show a very short lifetime in blood and accumulate in the liver and the spleen after intravenous administration [2]]. They are suitable carriers to target drugs to organs of the mononuclear phagocyte system (MPS) [3]. Their accumulation in the liver was explained by their recognition and uptake by macrophages of the MPS in relation with their strong capacity to activate the complement system $[3,4]$.

Capacity to activate the complement system and the more general phenomenon of opsonisation consisting in protein adsorption on nanoparticles in contact with blood depends strongly on nanoparticle surface properties. It is well known that these phenomena are affected by the nanoparticle size, the charge and the hydrophilicity of the nanoparticle surface [5-7]. More recently, it was reported that it also depends on the conformation of polysaccharide chains stranded on nanoparticle surface to achieve stability of nanoparticle dispersion $[4,8]$. The most unexpected result was an opposite capacity of activating the complement system reported with dextran-coated PACA-NPs of the same composition but obtained from two methods of polymerization. It was explained by a difference of the chain conformation arrangement in the coat of dextran due to the mechanism of polymerization used to produce the nanoparticles. Indeed, it was demonstrated that the polysaccharide chains form "loops" on the surface of PACA-NPs produced by AEP (AEP-PACA-NPs) while they form a dense "brush" on the surface of PACA-NPs produced by redox radical emulsion polymerization (RREP) (RREP-PACA-NPs) [4,8] (Figure 1). This last configuration of the dextran chains hampered adsorption of large proteins on the surface of nanoparticles including the protein $\mathrm{C} 3$ of the complement system [9]. It was found suitable to obtain nanoparticles with a low capacity to activate the complement system in contrast with the conformation found on the surface of the AEP PACA-NPs $[\mathbf{9 , 1 0}]$. All previous data suggested that RREP-PACA-NPs were a new drug carrier with distinct pharmacokinetic and biodistribution profile compared to those described for the well-known AEP-PACA NPs.

The aim of the present work was to investigate the pharmacokinetic and tissue distribution of a drug formulated with the RREP-PACA-NPs and to compare the results to those obtained for the free drug and for the drug associated with AEP-PACA-NPs. For this work, doxorubicin was chosen as the model drug because the pharmacokinetic and tissue distribution were 
already well known for the free drug and for the drug formulated in AEP-PACA-NPs. The association and stability of doxorubicin formulated as AEP PACA-NPs and RREP PACANPs were described and compared in a recent work [11]. Additionally, based on the knowledge available on these two nanoparticle formulations, we believed that they were relevant models to study the influence of the conformational characteristics of dextran in their corona on the pharmacokinetic and biodistribution of an associated drug.

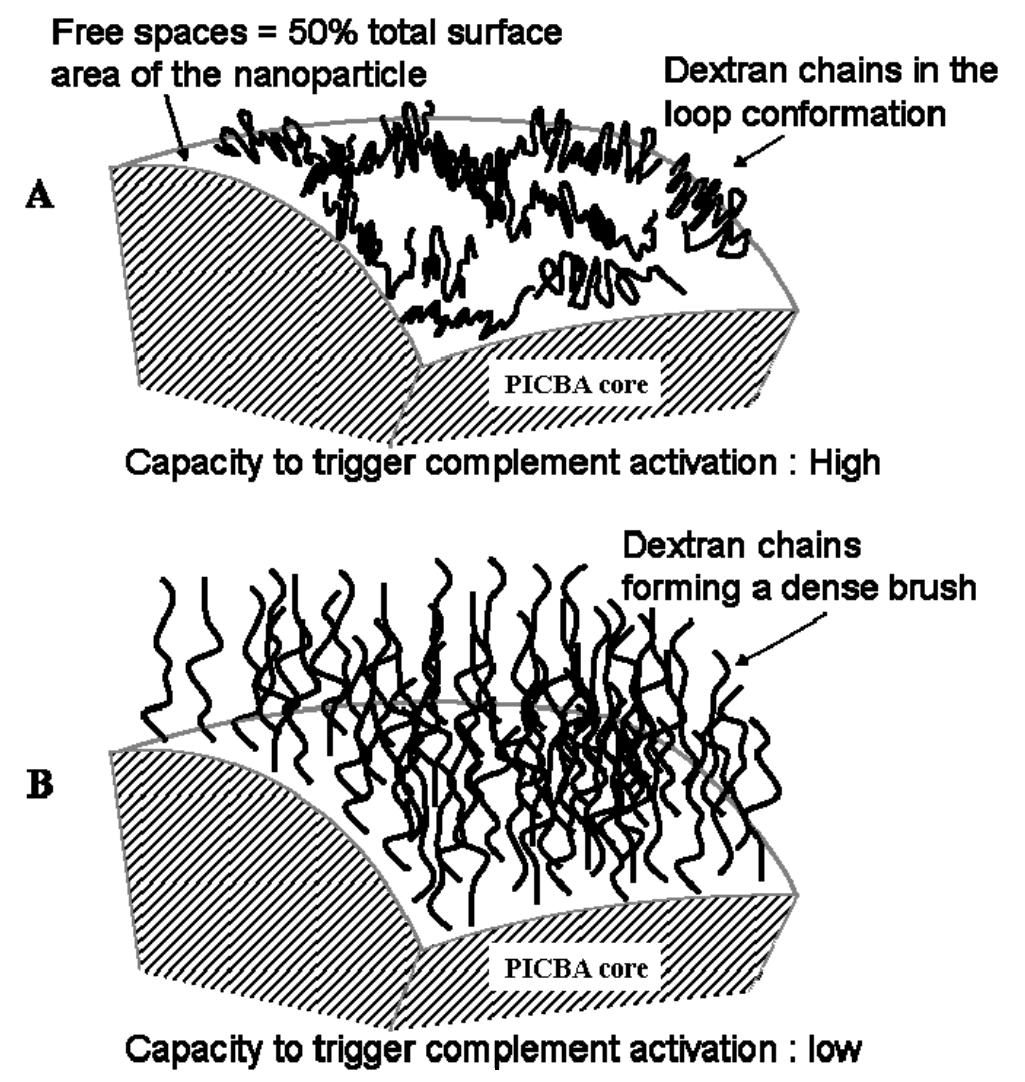

Figure 1: Scheme of the conformation taken by dextran chains at the surface of the PIBCA nanoparticles prepared by the AEP (AEP) and the RREP method (B) as deduced from previous works (Vauthier et al. 2009, 2011). The capacity of these nanoparticles to trigger the activation of the complement system was well correlated with the characteristics of the nanoparticle corona regarding their property to let large proteins such as the protein C3 of the complement system to diffuse across and to reach the nanoparticle core surface (Labarre et al. 2005). 


\section{Materials and Methods}

\subsection{Preparation of nanoparticles}

Dox-loaded RREP and AEP-PACA NP were prepared according to previously described methods (11). The mean hydrodynamic diameter of the Dox-loaded RREP-PACA-NPs was $301 \pm 82 \mathrm{~nm}(\mathrm{PDI}=0.3 \pm 0.05)$ and their zeta potential was $1.1 \pm 0.1 \mathrm{mV}$ as determined using a Malvern Zetasizer nano ZS90 (Malvern Instruments, Orsay, France). The mean hydrodynamic diameter and zeta potential of the Dox-loaded AEP-PACA-NPs were $196 \pm 48$ $\mathrm{nm}(\mathrm{PDI} 0.05 \pm 0.02)$ and. $0.7 \pm 0.1 \mathrm{mV}$, respectively.

\subsection{Pharmacokinetics and biodistribution study of the free Dox and the Dox associated with nanoparticles}

Animals: Experiments were performed on male Wistar rats (Janvier, Le Genest Saint Isle, France) weighing 240-300 g. Animals were stabilized under normal conditions for 1 week prior to experimentation and had free access to food and water. Experiments were carried out in compliance with the guidelines of the European Community (Recommendation 2007/ 526/ EC). The protocol ethics were institutionally approved.

Pharmacokinetics: Under general anesthesia by pentobarbital $(50 \mathrm{mg} / \mathrm{kg} \mathrm{bw})$, a catheter was introduced into the rat jugular vein, positioned subcutaneously with the tip in the inter scapular region. The rat was allowed to recover all night, and the blood catheter was flushed with heparinized saline solution $(\mathrm{NaCl} 0.9 \%$, heparin $50 \mathrm{UI} / \mathrm{ml})$. The three formulations, free Dox (f-Dox), Dox loaded-AEP PACA-NPs (Dox-AEP) and Dox loaded-RREP PACA NPs (Dox-RREP), were administered at the dose of $6 \mathrm{mg} / \mathrm{kg}$ to three groups of 5 rats each. All formulations were administered in the non catheterized jugular vein by slow perfusion $(0.25$ $\mathrm{mL} / \mathrm{min}$ ). During the perfusion, the animal was maintained under isoflurane anesthesia (1\% of isoflurane in air). Blood samples were collected at time 10, 15, 30, $45 \mathrm{~min}$ and 1, 2, 4, 6, 10 and $24 \mathrm{~h}$, after administration. After each collection, the volume of the collected sample was replaced by the same volume of $0.9 \% \mathrm{NaCl}$ solution. Immediately after collection, blood was centrifuged (2000 g for $10 \mathrm{~min}$ ) to recover plasma samples which were then stored at $-20{ }^{\circ} \mathrm{C}$ until analysis.

Biodistribution: To study tissue biodistribution, 45 rats were randomly divided in three groups of 15 rats. Each group received an injection of one of the three formulations in the jugular vein. At time 1, 4, 10, 24 and $48 \mathrm{~h}$ after dosing, three rats of each group were sacrificed by 
cardiac puncture under general pentobarbital anesthesia. Heart, lungs, liver, kidneys, spleen and brain were collected and stored at $-20{ }^{\circ} \mathrm{C}$ until analysis. Concentration in Dox in each organ was analyzed in duplicate.

\subsection{Dox analysis in plasma and tissues}

Dox analyses in plasma and tissue were performed using a HPLC method after liquid-liquid extraction as reported elsewhere (12)

\subsection{Pharmacokinetic analysis and statistics}

Statistical studies were made using $t$ tests of unequal variance performed by Origin 8 software, (Origin Lab corp., USA). For pharmacokinetic analysis, the plasma concentrations of each rat were analyzed with the WinNonlin ${ }^{\circledR}$ professional version 5.2 software (Pharsight, Sunnyvale, CA, USA), using a bicompartmental model. The estimation method was the weighted last squares (WLS) weighting by the inverse of the variance $1 / \sigma^{2}$. The distribution $(\alpha)$ and elimination $(\beta)$ rate-constants and the maximal concentrations $\mathrm{A}$ and $\mathrm{B}$ were determined by nonlinear regression of the observed data. The area under the plasma concentration-versus-time curve (AUC) and the area under the first moment curve (AUMC) were determined by the equations (1) and (2), respectively.

$\mathrm{AUC}_{0-\infty}=\mathrm{A} / \alpha+\mathrm{B} / \beta$

$\mathrm{AUMC}_{0-\infty}=\mathrm{A} / \alpha^{2}+\mathrm{B} / \beta^{2}$

Total clearance $(\mathrm{CL})$ was calculated from the ratio dose/ $/ \mathrm{AUC}_{0-\infty}$. The steady state volume $\left(V \mathrm{~d}_{\mathrm{ss}}\right)$ was calculated from $\left(\mathrm{dose}^{*} \mathrm{AUMC}_{0-\infty} /\left(\mathrm{AUC}_{0-\infty}\right)^{2}\right)$. The distribution and elimination half-lives $\left(t_{1 / 2 \alpha}\right.$ and $\left.t_{1 / 2 \beta}\right)$ were calculated from $\operatorname{Ln} 2 / \alpha$ and $\mathrm{Ln} 2 / \beta$, respectively.

\section{RESULTS AND DISCUSSION}

\subsection{Pharmacokinetics:}

Figure 2A reported the mean Dox plasma concentration as a function of time following intravenous administration of the three formulations. The Dox plasma concentration-time profiles showed biphasic curves with a first rapid distribution phase followed by a slow elimination phase. Each formulation of Dox showed a single pharmacokinetic profile 
indicating that the drug remained associated with the nanoparticles and followed the in vivo fate of the nanocarrier. Individually pharmacokinetic curves can be well fitted with a bicompartmental model of pharmacokinetics (Figure 2B).

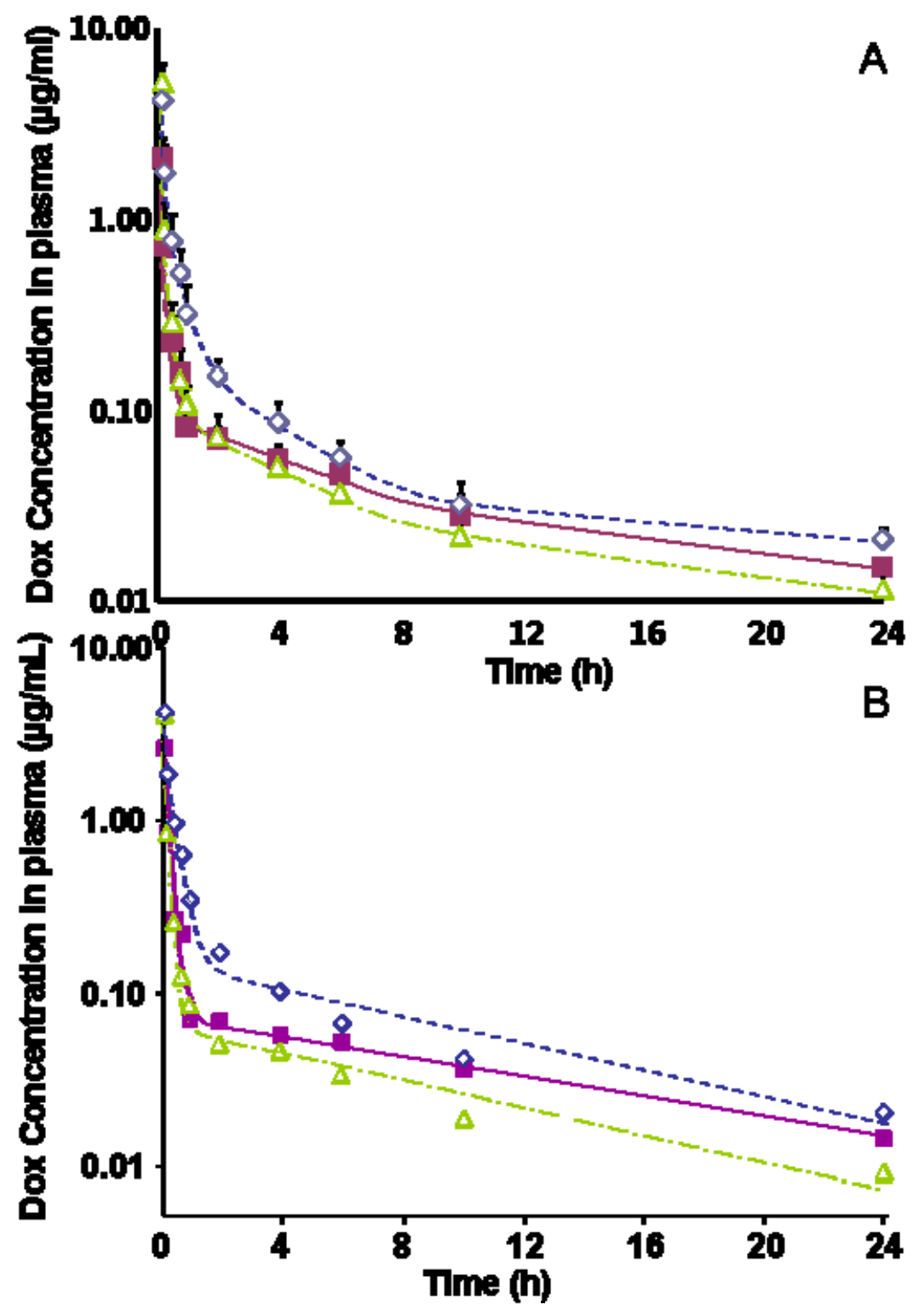

Figure 2: Pharmacokinetics of doxorubicin after intravenous administration of different formulations to rats. (A) Data are given as the mean concentration of doxorubicine in plasma of rats $(n=5)$. (Full square): free Dox (f-Dox), (empty diamond): Dox loaded RREP PACA-NPs (Dox-RREP), (empty triangle): Dox loaded AEP PACA-NPs (Dox-AEP). Lines only serve as guides for the eyes. (B) Example of the analysis of the pharmacokinetic data using a bicompartmental model performed on one animal of each group having received a different formulation of Dox by the intravenous route. Experimental data were given as the symbols on the graph: (full square): free Dox (f-Dox), (empty diamond): Dox loaded RREP PACA-NPs (Dox-RRP) and (empty triangle): Dox loaded AEP PACA-NPs (Dox-AEP). Curves were drawn from the predicted values calculated on the basis of the assumption of a bi-compartmental pharmacokinetic model with the help of the WinNinLin software: (-) f-Dox, ( - - ) Dox-RREP, ( _._- ) Dox-AEP. 
The concentrations of Dox-RREP were significantly higher than those of f-Dox and Dox-AEP $(p<0.05)$ at the distribution phase. While there was no significant difference between DoxAEP and f-Dox in the elimination phase until 10 hours, there was a significant difference between Dox-RREP and Dox-AEP. Twenty four hours after administration, the difference between Dox-RREP and the other formulations was significant. Table 1 summarizes the main pharmacokinetic parameters calculated using a bi-compartmental pharmacokinetic analysis. The main difference between the three formulations was in the distribution phase. The DoxRREP showed the longer distribution phase compared with the other formulations. The distribution half-life $\left(t_{1 / 2 \alpha}\right)$ of Dox-RREP was about twice that of f-Dox (Table $1 ; p=0.001$ ) and 3.5 fold higher than that of Dox-AEP $(p=0.0001)$. As compared to f-Dox, a faster distribution rate was obtained with the AEP-NPs $(p=0.042)$ while a slower one was obtained with the RREP-NPs $(p=0.001)$. Results obtained with Dox-AEP can be explained by the rapid uptake of the AEP-NPs by the macrophages of the mononuclear phagocyte system (MPS) in agreement with previous reports $[\mathbf{2 , 3 , 1 3}$. Results obtained with the Dox-RREP were in agreement with the low capacity of the RREP-NPs to trigger the activation of the complement system reported earlier [4,9].

The increase of blood circulation time of Dox by RREP nanoparticles was also evidenced by the increase of $\mathrm{AUC}_{0-\infty}$ value and the decrease of the total clearance $(\mathrm{Cl})$ in comparison with $\mathrm{f}-$ Dox and Dox-AEP (Table 1; $p=0.003,0.009,0.023$ and 0.029, respectively).

Table 1: Pharmacokinetic parameters of Dox after i.v. administration to rats in different formulations: free Dox (f-Dox), Dox loaded RREP PACA-NPs (Dox-RREP) and Dox loaded AEP PACA -NPs (Dox-AEP). ( $n=5)$, * $p<0.05$ Dox-RREP vs $f$-Dox, ${ }^{*} p<0.05$ Dox-RREP vs Dox-AEP, ${ }^{¥} p<0.05$ Dox-AEP vs $f$-Dox)

\begin{tabular}{|l|l|l|l|}
\hline Parameter & f-Dox & Dox-AEP & Dox-RREP \\
\hline$t_{1 / 2 \square \square}(\mathrm{h})$ & $0.11 \pm 0.03$ & $0.06 \pm 0.03^{¥}$ & $0.21 \pm 0.03^{* \#}$ \\
$t_{1 / 2 \square \square}(\mathrm{h})$ & $9.69 \pm 1.09$ & $7.26 \pm 2.09$ & $8.56 \pm 0.71$ \\
$\mathrm{AUC}_{0-\infty}(\mathrm{h} . \mu \mathrm{g} / \mathrm{mL})$ & $1.45 \pm 0.21$ & $1.59 \pm 0.40$ & $2.50 \pm 0.43^{\text {*\# }}$ \\
$\mathrm{Cl}(\mathrm{L} / \mathrm{h})$ & $1.14 \pm 0.32$ & $1.03 \pm 0.27$ & $0.65 \pm 0.09^{\text {*\# }}$ \\
$\mathrm{Vss}(\mathrm{L})$ & $12.0 \pm 4.84$ & $6.96 \pm 1.89$ & $4.98 \pm 1.76^{*}$ \\
& & & \\
& & & \\
\hline
\end{tabular}


The steady state volume of distribution (Vss) of Dox-RREP was significantly smaller than that of f-Dox $(p=0.028)$ that showed a less extensive distribution of Dox in tissue when it was administered with RREP-NPs. In contrast, there was no difference between the Vss of Dox-RREP and Dox-AEP as well as between Dox-AEP and f-Dox.

The pharmacokinetics parameters of Dox-loaded RREP nanoparticles showed that these nanoparticles behaved like stealth nanoparticles. Compared to f-Dox or Dox-AEP our results show that Dox-RREP has a higher distribution half-life and a higher AUC with a slower clearance. Although the concentrations of Dox during the elimination phase were significantly higher in the Dox-RREP treated rats, there was no significant difference between the elimination half-life $\left(t_{1 / 2 \beta}\right)$ between the three formulations. This result suggested that the elimination of Dox concerned only the free fraction of the drug.

\subsection{Tissue distribution}

Figure 3 reported Dox concentrations found in different organs of the rats after intravenous administration of the three types of Dox formulations. As shown on this figure, significant amount of Dox was found in the different organs including the liver, spleen, heart, lungs and kidneys. The concentrations varied with time and were greatly influenced by the type of injected formulation. No Dox was found in the brain of rats treated by the different formulations.

This confirmed that Dox followed the biodistribution of the drug carrier with which it was associated. Dox concentrations found in liver of rats treated with Dox-RREP were significantly lower than that found in animals of the group treated with Dox-AEP. It is noteworthy that this was observed over the whole period of time of the pharmacokinetic experiment which lasted for 48 hours. These results agreed with those of the pharmacokinetic study and indicated a lower uptake of Dox by the MPS when it was associated with the RREP-PACA-NPs as compared to Dox-AEP and f-Dox. Expressed as the percentage of the dose administered, the amount of Dox found in the liver after 1 hour of the administration of Dox-RREP $(5 \pm 1 \%)$ was two times lower than that found following the administration of Dox-AEP $(10 \pm 2 \%)$ and the f-Dox $(9 \pm 1 \%)$. Concentrations of Dox found in the spleens were similar for the three formulations at all studied time points (figure 3.b). The group of rats treated with Dox-RREP showed significantly higher concentrations of Dox in the heart and lungs during the first $4 \mathrm{~h}$ of the experiment than those observed in groups treated with f-Dox and Dox-AEP (figure 3.c, 3.d). For instance, the fractions of the dose which reached these organs with the Dox-RREP formulation were $1.8 \pm 0.4 \%$ in the heart and $1.1 \pm 0.2$ in the 
lungs one hour after the injection while it was $8 \pm 2 \%$ at the same time post injection in the kidneys. In comparison, these values were twice to three times lower considering the DoxAEP (heart: $0.7 \pm 0.1 \%$, lungs: $0.63 \pm 0.06 \%$, kidneys: $2.5 \pm 0.4 \%$ ) and the f-Dox (heart: $0.8 \pm$ $0.2 \%$, lungs: $0.6 \pm 0.1 \%$, kidneys: $2.9 \pm 0.5 \%$ ) formulations. As the Dox administered under the form of RREP-PACA-NPs were less cleared by the liver they can reach other organs at higher concentrations compared to Dox administered under the free form and under the form of AEP-PACA-NPs. The Dox concentration in kidneys of Dox-RREP treated rats were significantly higher than those of two other formulations (figure 3.e) while no significant differences in Dox concentrations were observed between the f-Dox and Dox-AEP treated groups.
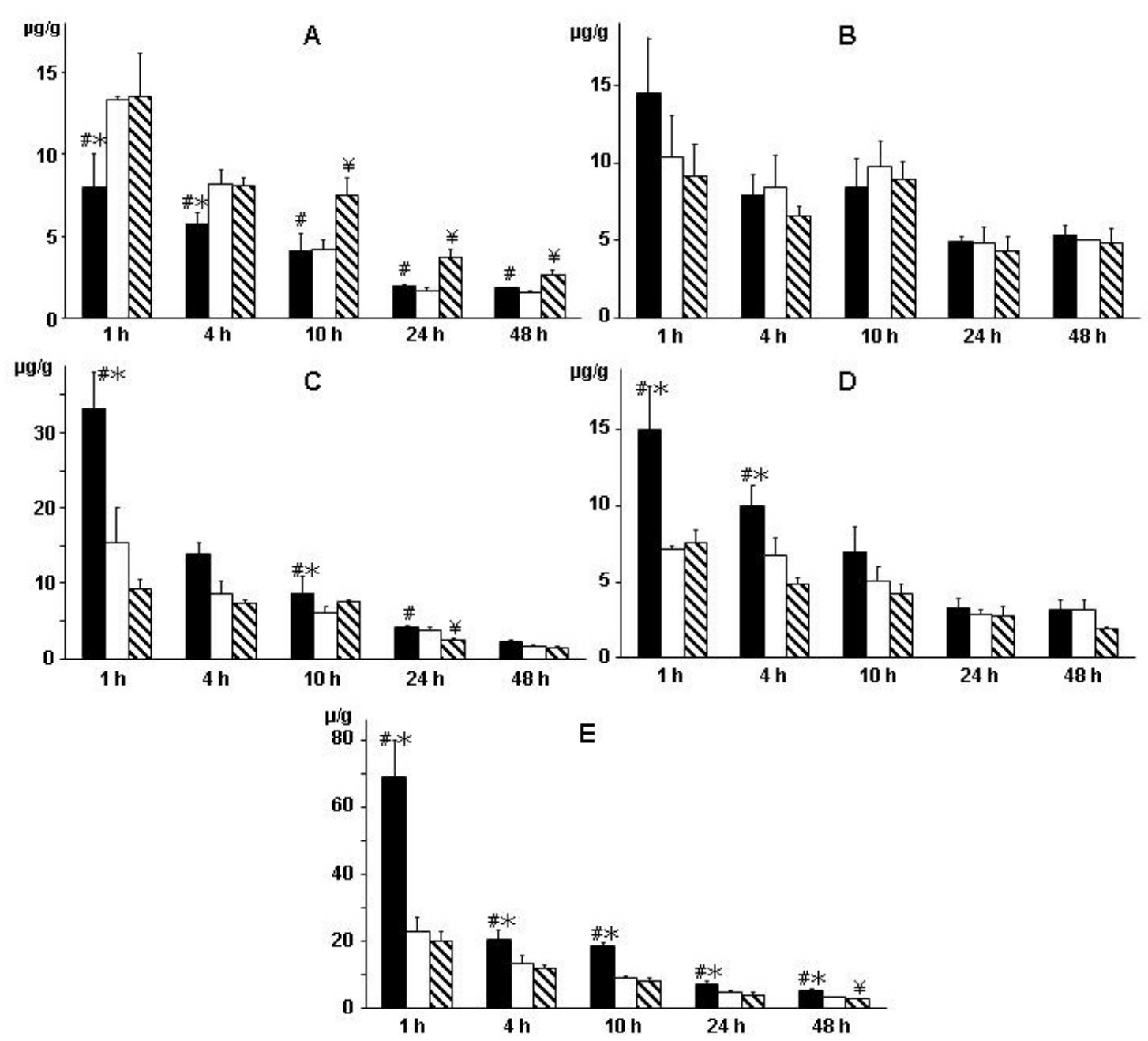

Figure 3: Body distribution of doxorubicin after intravenous administration of different formulations, free Dox (f-Dox) (white columns), Dox loaded RREP PACA-NPs (DoxRREP) (black columns) and Dox loaded AEP PACA-NPs (Dox-AEP) (hashed columns), to rats. Concentrations of doxorubicin in organs ( $y$ axis) were determined in different organs: liver (A), spleen (B), heart (C), lungs (D) and kidneys (E) at different times ( $x$ axis). Statistics: *: $p<0.05$ Dox-RREP vs f-Dox, \#: $p<0.05$ Dox-RREP vs Dox-AEP, ¥: $p<$ $0.05 f-D o x$ vs Dox-AEP, $n=3$. 
The biodistribution of Dox found with the formulation Dox-AEP agreed with results reported in previous works $(\mathbf{2 , 3 , 1 3})$. It confirmed the rapid uptake of the Dox-AEP by macrophages of the MPS and possibility to target the associated drug in the liver. Results from both the pharmacokinetics and the biodistribution study of the Dox-RREP formulation supported the hypothesis that the RREP-PACA NPs were stealth and agreed with their low capacity to activate the complement system reported earlier $(\mathbf{4 , 9})$. However, based on the criteria of the size of the nanoparticles, results were in contrast with those expected from the literature (7). Indeed, the larger nanoparticles (PPER-PACA NPs) appeared stealth while it was expected to be highly susceptible to recognition mechanisms of the defense systems of the organisms because of their large size. Since all experimental results were in agreement with each others and with previous findings, it can be concluded that surface properties of nanoparticles were predominant parameters to determine the in vivo fate of the present nanoparticles. Thus, the difference in the pharmacokinetics and biodistribution of Dox highlighted between the two nanoparticle formulations considered in the present work can be attributed to their difference in the conformation of the dextran chains decorating the surface of the nanoparticles. It is noteworthy, that uptake of foreign bodies by the spleen occurred irrespectively of their surface properties. A similar fate was reported by other authors considering PACA-NPs decorated with poly(ethylene-glycol) chains $[\mathbf{1 4 , 1 5}]$. It can be suggested that a different mechanism of recognition was involved in the spleen compared to that used by liver macrophages.

In conclusion, results from this study showed that conformation of polysaccharide chains decorating the nanoparticle surface are influencing the pharmacokinetic and biodistribution of nanoparticles. The stealth property described for the first time for the RREP-NPs was different from the know stealth property described for pegylated nanoparticles which generally show a much longer time of persistence in the blood circulation during the distribution phase of the pharmacokinetic $[\mathbf{6 , 1 4 , 1 5}]$. The rather short half life in the blood circulation was explained by the rapid accumulation of the nanoparticles in organs which occurred in parallel with the reduction of the plasma concentration of the drug. Thus, the RREP-NPs are stealth with a short circulation time. By analogy of the accumulation of the drug carried by the RREP nanoparticles in highly vascularised tissued, it might be expected that these nanoparticles may enhance drug delivery in tumors thanks to the enhanced permeation and retention effect. In contrast, the delivery of drugs in the brain will probably be 
hampered as suggested by the total absence of distribution of Dox in this organ and because of the rapid clearance from the blood compartment.

\section{Acknowledgments}

The authors wish to thank Henkel Biomedical for having provided the monomer used to synthesize the nanoparticles used in this study. K.A. thanks the Ministry of Health of Syria for providing with a Ph.D. scholarship. We thank Pharsight Corporation (Sunnyvale, CA, USA) for allowing our University to benefit from the Academic Licensing Program.

\section{Declaration of Interest}

The authors report no declarations of interest.

\section{References}

[1] C. Vauthier, D. Labarre, G. Ponchel, Design aspects on poly(alkylcyanoacrylate) nanoparticles for targeted drug delivery, J. Drug Targeting 15 (2007) 641-663.

[2] L. Grislain, P. Couvreur, V. Lenaerts, M. Roland, D. Deprez-Decampeneere, P. Speiser, Pharmacokinetics and distribution of a biodegradable drug-carrier, Int. J. Pharm. 15 (1983) 335-345.

[3] N. Chiannilkulchai, N. Ammoury, B. Caillou, J.-P. Devissaguet, P. Couvreur, Hepatic tissue distribution of doxorubicin-loaded nanoparticles after i.v. administration in reticulosarcoma M 5076 metastasis-bearing mice, Cancer Chemother. Pharmacol. 26 (1990) 122-126.

[4] D. Labarre, C. Vauthier, C. Chauvierre, B. Petri, R. Muller, M.-M. Chehimi, Interactions of blood proteins with poly(isobutylcyanoacrylate) nanoparticles decorated with a polysaccharidic brush, Biomaterials 26 (2005) 5075-5084.

[5] S.M. Moghimi, A.C. Hunter, J.C. Murray, Nanomedicine: current status and future prospects, FASEB Journal 19 (2005) 311-330.

[6] A. Vonarbourg, C. Passirani, P. Saulnier, P. Simard, J.-C. Leroux, J.-P. Benoit, Evaluation of pegylated lipid nanocapsules versus complement system activation and macrophage uptake, J. Biomed. Mater. Res. A. 78 (2006) 620-628.

[7] F. Alexis, E. Pridgen, L.-K. Molnar, O.-C. Farokhzad, Factors affecting the clearance and biodistribution of polymeric nanoparticles, Mol. Pharm. 5 (2008) 505-515. 
[8] I. Bertholon, C. Vauthier, D. Labarre, Complement activation by core-shell poly(isobutylcyanoacrylate)-polysaccharide nanoparticles: influences of surface morphology, length, and type of polysaccharide, Pharm. Res. 23 (2006) 1313-1323.

[9] C. Vauthier, B. Persson, P. Lindner, B. Cabane, Protein adsorption and complement activation for di-block copolymer nanoparticles, Biomaterials 32 (2011) 1646-1656.

[10] C. Vauthier, P. Lindner, B. Cabane, Configuration of Bovine Serum Albumin adsorbed on polymer particles with grafted dextran corona, Coll. \& Surf. B 69 (2009) 207-215

[11] K. Alhareth, C. Vauthier, C. Gueutin, G. Ponchel, F. Moussa, Doxorubicin loading and in vitro release from poly(alkylcyanoacrylate) nanoparticles produced by Redox Radical Emulsion Polymerization, J. Appl. Polym. Sci. 119 (2011) 816-822.

[12] K. Alhareth, C. Vauthier, C. Gueutin, G. Ponchel, F. Moussa, HPLC quantification of doxorubicin in plasma and tissues of rats treated with doxorubicin loaded poly(alkylcyanoacrylate) nanoparticles. J. Chrom. B. 887- 888 (2012) 128- 132.

[13] L.H. Reddy, R.S.R. Murthy, Pharmacokinetics and biodistribution studies of doxorubicin loaded poly(butylcyanoacrylate) nanoparticles synthesized by two different techniques. Biomed. Papers 148 (2004) 161-166.

[14] M.-T. Peracchia, E. Fattal, D. Desmaële, M. Besnard, J.-P. Noël, J.-M. Gomis, M. Appel, J. d'Angelo, P. Couvreur, Stealth PEGylated polycyanoacrylate nanoparticles for intravenous administration and splenic targeting, J. Control. Release 60 (1999) 121-128

[15] P. Calvo, B. Gouritin, H. Chacun, D. Desmaële, J. D'Angelo, J.-P. Noel, D. Georgin, E. Fattal, J.-P. Andreux, P. Couvreur, Long-circulating PEGylated polycyanoacrylate nanoparticles as new drug carrier for brain delivery, Pharm. Res. 18 (2001) 1157-1166. 